Cristiani Garrido de Andrade ${ }^{1}$

Solange Fátima Geraldo da Costa ${ }^{1}$

Maria Emília Limeira Lopes ${ }^{1}$

${ }^{1}$ Departamento de

Enfermagem, Centro de Ciências da Saúde, Campus I, Universidade Federal da Paraíba. Cidade

Universitária - Campus I, Castelo Branco. 58.059-900 João Pessoa PB.

cristiani_garrido@hotmail.com

\title{
Cuidados paliativos: a comunicação como estratégia de cuidado para o paciente em fase terminal
}

\author{
Palliative care: \\ communication as a strategy of care for the terminal patient
}

\begin{abstract}
Palliative care involves an approach in the field of care for terminal patients and their families that seeks to assure them better quality of life by establishing good communication. The scope of this study was to verify how nurses use communication in the field of palliative care when assisting patients in the terminal phase. This is exploratory research of a qualitative nature in which 28 nurses working in wards of a hospital in the city of Joao Pessoa in the State of Paraíba participated in the period from August to October 2012. A form was used for data collection that was then analyzed using the content analysis technique. Three categories emerged from the analysis of the material: "palliative care and communication - interpersonal relationship between the nurse and the terminal patient"; "communication in palliative care as a strategy for strengthening the bond between the nurse and the terminal patient"; and "the importance of communication between the nurse and the family of the terminal patient under palliative care." The conclusion reached was that communication is seen to be an effective element of care for the patient in the terminal phase and it is extremely important for the promotion of palliative care.
\end{abstract}

Key words Communication, Palliative care, Nursing
Resumo Os cuidados paliativos compreendem uma abordagem de assistência ao paciente sem possibilidades de cura e sua família, com o objetivo de proporcionar-lhes uma melhor qualidade de vida, a partir, essencialmente, de uma boa comunicação, sobretudo no campo do cuidado ao paciente terminal. O estudo teve como objetivo averiguar como enfermeiros utilizam a comunicação, no âmbito dos cuidados paliativos, ao assistir o paciente em fase terminal. Trata-se de uma pesquisa exploratória, de natureza qualitativa, na qual participaram 28 enfermeiros atuantes em unidades de internação de um hospital da cidade de João Pessoa (PB), no período de agosto a outubro de 2012. Para a coleta dos dados utilizou-se um formulário. Os dados foram analisados mediante a técnica de análise de conteúdo. Da análise do material, emergiram três categorias: "cuidados paliativos e comunicação - relação interpessoal do enfermeiro com o paciente terminal"; "comunicação em cuidados paliativos como estratégia para fortalecimento do vínculo entre enfermeiro e paciente terminal"; e "importância da comunicação entre enfermeiro e família do paciente terminal sob cuidados paliativos." Conclui-se que a comunicação se configura como um elemento eficaz do cuidado com o paciente em fase terminal e é de suma importância para a promoção dos cuidados paliativos.

Palavras-chave Comunicação, Cuidados paliativos, Enfermagem 


\section{Introdução}

A filosofia dos cuidados paliativos iniciou-se na Inglaterra, em 1967, a partir da iniciativa de Cicely Mary Strode Saunders (assistente social, enfermeira e médica) que disseminou essa nova maneira de cuidar aos pacientes que vivenciavam a terminalidade e a proximidade com a morte ${ }^{1}$. Tais cuidados visam a compreender todas as necessidades do paciente (dentro dos limites possíveis), contemplando-o como um ser integral ${ }^{2}$.

É notório destacar que o vocábulo paliativo deriva do latim pallium, que significa manto. Tal terminologia denota a ideia principal dessa filosofia: de proteger, amparar, cobrir, abrigar, quando a cura de determinada doença não é mais possível. Além disso, no latim, pallium são vestimentas usadas pelo Papa, portanto, há uma forte ligação desse termo histórico com o sagrado e com a espiritualidade ${ }^{1,3}$.

Dessa forma, os cuidados paliativos constituem um campo interdisciplinar de cuidados totais, ativos e integrais, destinados a melhorar a qualidade de vida do paciente sem possibilidades de cura e dos seus familiares, por meio de avaliação correta e de tratamento adequados para o alívio da dor e dos sintomas decorrentes da fase avançada de uma doença, além de proporcionar suporte psicossocial e espiritual, em todos os estágios, desde o diagnóstico de uma doença incurável até o período de luto da família4.

Assim sendo, é inegável a valorização dos cuidados paliativos direcionados ao paciente na terminalidade de vida, bem como de diversas estratégias de cuidar utilizadas nesta modalidade, dentre as quais destaca-se a comunicação.

A comunicação é intrínseca ao comportamento humano e permeia todas as suas ações no desempenho de suas funções. Etimologicamente, a palavra comunicar origina-se do latim communicare, que significa pôr em comum. Assim, a comunicação pode ser compreendida como uma técnica de trocas e de compreensão de mensagens, emitidas e recebidas, mediante as quais as pessoas se percebem e partilham o significado de ideias, pensamentos e propósitos ${ }^{3}$.

No âmbito da Enfermagem, a comunicação representa uma estratégia de suma relevância para a prática dos cuidados paliativos. E quando subsidiada por uma relação de atitude, cooperação, sentimento e sensibilidade, este instrumento é um importante impulsionador da relação entre o enfermeiro e o paciente em fase terminal ${ }^{5}$.

Vale salientar que a comunicação vai muito além das palavras e do conteúdo, uma vez que contempla a escuta atenta, o olhar e a postura, porquanto o emprego eficaz desse recurso é uma medida terapêutica comprovadamente eficiente para pacientes que dele necessitam, sobretudo os que se apresentam em fase terminal ${ }^{6}$.

Nessa perspectiva, a comunicação adequada é considerada um método fundamental para o cuidado integral e humanizado porque, por meio dela, é possível reconhecer e acolher, empaticamente, as necessidades do paciente, bem como de seus familiares. Quando o enfermeiro utiliza esse recurso de forma verbal e não verbal, permite que o paciente possa participar nas decisões e cuidados específicos relacionados com a sua doença e, dessa forma, obtenha um tratamento digno ${ }^{5}$.

Trata-se, pois, de um aspecto fundamental para intermediar as relações humanas, promover a sustentabilidade e a consolidação da autonomia, diante das perspectivas individuais, e traduz-se como um elemento diagnóstico e terapêutico, capaz de identificar demandas assistenciais e acolher terapeuticamente, proporcionando fortes vínculos dos enfermeiros com os pacientes na finitude de vida e com sua família ${ }^{7}$.

Diante do exposto, considerando a relevância da temática na área e o quantitativo ínfimo de estudos acerca dos cuidados paliativos e da comunicação em relação ao cuidado com o paciente terminal na literatura nacional, emergiu o interesse em desenvolver este estudo que teve como fio condutor o seguinte questionamento, também seu objetivo: como enfermeiros utilizam a comunicação, no âmbito dos cuidados paliativos, ao assistir o paciente em fase terminal?

\section{Métodos}

Trata-se de uma pesquisa exploratória, de natureza qualitativa. O cenário da investigação foram as unidades de internação de um hospital público, localizado na cidade de João Pessoa (PB), considerado como de referência nesse Estado.

Os participantes da pesquisa foram 28 enfermeiros assistenciais da instituição selecionada para o estudo, que prestam cuidados direcionados ao paciente em fase terminal, selecionados mediante os seguintes critérios: que o profissional atuasse há pelo menos um ano na referida unidade, estivesse em atividade profissional durante o período de coleta de dados e tivesse disponibilidade e interesse para participar da pesquisa, confirmando sua concordância com a assinatura do Termo de Consentimento Livre e Esclarecido. A coleta de 
dados ocorreu durante o período de agosto a outubro de 2012 e somente foi iniciada após a aprovação do projeto de pesquisa pelo Comitê de Ética em Pesquisa do Hospital Universitário Lauro Wanderley da Universidade Federal da Paraíba (HULW/UFPB). Dessa forma, ressalte-se que o estudo foi realizado considerando-se as observâncias éticas contempladas na Resolução 196/ $96^{8}$ do Conselho Nacional de Saúde, no que concerne às normas e às diretrizes regulamentadoras da pesquisa com seres humanos.

Para a obtenção do material empírico, foi utilizado um formulário com questões pertinentes ao objetivo proposto: Qual a importância da comunicação na prática dos cuidados paliativos no que concerne à assistência de enfermagem direcionada ao paciente em fase terminal? Como você utiliza a comunicação na prática dos cuidados paliativos para assistir o paciente em fase terminal? Convém mencionar que, para manter o anonimato dos enfermeiros inseridos no estudo, os depoimentos oriundos do referido formulário foram identificados pela sigla "Enf., seguido de números de um a vinte e oito. Exemplo: o primeiro enfermeiro entrevistado foi codificado da seguinte maneira: "Enf.1"; o segundo profissional, "Enf.2" e assim por diante.

Os dados obtidos através dos instrumentos propostos foram analisados qualitativamente, mediante a análise de conteúdo proposta por Bardin ${ }^{9}$, definida como conjunto de técnicas de análise das comunicações visando obter, por procedimentos sistemáticos e objetivos de descrição do conteúdo das mensagens, indicadores que permitam a inferência de conhecimentos relativos às condições de produção/recepção destas mensagens.

Esta seguiu as seguintes etapas: pré-análise, que consistiu em organizar os dados coletados por meio dos formulários; exploração do material, identificando-se os pontos relevantes de cada questão, com seus respectivos pontos convergentes de acordo com o seu foco comum para, depois, agrupá-los em categorias e tratar os resultados, momento em que foram abordadas as inferências e as interpretações ${ }^{9}$.

Da análise, emergiram as seguintes categorias: "Cuidados paliativos e comunicação - relação interpessoal do enfermeiro com o paciente terminal"; "Comunicação em cuidados paliativos como estratégia para fortalecimento do vínculo entre enfermeiro e paciente terminal"; $e^{\text {"Im- }}$ portância da comunicação entre enfermeiro e família do paciente terminal sob cuidados paliativos".

\section{Resultados e Discussão}

Neste estudo foram contempladas três categorias temáticas cujo conteúdo desvela como os enfermeiros utilizam a comunicação para humanizar o cuidar em enfermagem para o paciente em fase terminal e sua família, com ênfase na valorização da comunicação verbal e não verbal, pautadas nos cuidados paliativos.

\section{Cuidados paliativos e comunicação: relação interpessoal do enfermeiro com o paciente terminal}

No que diz respeito a essa categoria, os participantes resgatam a importância da relação humana, mostrando que a comunicação com o paciente que vivencia o processo de terminalidade é considerada o alicerce para um bom relacionamento interpessoal, como mostram estes discursos:

A comunicação é uma ferramenta extremamente relevante no processo de cuidar, principalmente quando se trata de paciente terminal, no sentido de fortalecer o vínculo entre paciente/profissional, estimular o paciente a verbalizar anseios, preocupações e dúvidas acerca da situação clínica, dar oportunidade ao paciente/familiar de verbalizar preferências no atendimento e ajudálos na tomada de decisões. (Enf.3)

[...] a comunicação traz tranquilidade e confiança com a equipe que o está acompanhando, fazendo com que o paciente se sinta a vontade em falar e expressar suas angústias, medos e ansiedades, ajudando no fortalecimento do vínculo. (Enf.8)

A comunicação é um elemento essencial na relação humana, através dela podemos detectar problemas, facilitar o alivio dos sintomas, estimular e melhorar a autoestima do paciente, conhecer valores, favorecer o bem-estar e detectar as necessidades dos pacientes. (Enf.17)

Mesmo que não seja um paciente que verbali$z a$, é importante proporcionar confiança, fazer com que ele saiba que tem um profissional que demonstra atenção, afeto e compromisso com o doente. (Enf.23)

O paciente só permite ser cuidado quando ele sente segurança no profissional que está the assistindo, e essa segurança só se dar através da comunicação, conversando com ele. (Enf.20)

Diante dos trechos dos depoimentos supracitados, os enfermeiros destacam a relevância da comunicação entre o enfermeiro e o paciente na terminalidade, visto que a condição em que ele se encontra coloca-o em situações que, às vezes, são 
difíceis de compreender, por serem caracterizadas pela dor e pelo sofrimento, que geram angústia e depressão. Portanto, a comunicação é uma das habilidades que devem ser empregadas pelo enfermeiro, para que ele possa expandir a habilidade de apreender as mensagens - implícitas ou explícitas - que permeiam sua relação com o paciente e os familiares.

No âmbito dos cuidados paliativos, a comunicação realizada de forma adequada é considerada como um pilar fundamental para a implementação de tal prática. Trata-se de um suporte que o paciente pode empregar para expressar seus anseios. Para isso, precisa de um cuidado integral e humanizado, que só é possível quando o profissional recorre às suas habilidades de comunicação, essencialmente, com o paciente em fase terminal, para estabelecer uma relação efetiva com ele ${ }^{10}$.

Verifica-se que o paciente em fase terminal, deseja ser compreendido como um ser humano que sofre, porque, além da dor física, passa por conflitos existenciais e necessidades que os fármacos ou os aparelhos de alta tecnologia não podem prover. Assim, além de compartilhar seus medos e anseios relacionando-se com seus pares, através da comunicação, ele necessita sentirse cuidado, amparado, confortado e compreendido pelos enfermeiros. Expressões de compaixão e de afeto na relação com o paciente trazem a certeza de que ele é parte importante de um conjunto, o que ocasiona sensação de proteção, de consolo e de paz interior ${ }^{11}$.

No momento em que o profissional se comunica com o paciente que vivencia a terminalidade de maneira adequada, fortalece o vínculo, adquire confiança e quase sempre, consegue decifrar informações essenciais e amenizar-lhe a ansiedade e a aflição ${ }^{12}$. Ressalta-se, então, que a comunicação se for explanada de maneira compreensível, ao paciente em fase terminal, contribui para que ele tenha consciência de sua dignidade durante toda a assistência prestada e lhe proporciona autonomia, quando precisa tomar decisões sobre sua vida e seu tratamento ${ }^{13}$.

A literatura esclarece ${ }^{13}$ que o enfermeiro ao prestar assistência ao paciente de forma holística, fazendo o uso da comunicação como uma ferramenta para o estabelecimento de uma relação de confiança, atende as suas necessidades e se fortalece diante do enfrentamento de perdas, de doença, de incapacidades e de morte. Esse fortalecimento incide do resultado do cuidado, pela promoção do conforto, do alívio da dor e da preservação da autoestima do paciente. Portanto, ao estabelecer relação com o paciente, com base na confiança, o enfermeiro favorece um vínculo de cuidado com ele, que embasa o relacionamento interpessoal, tem a sensação de missão desempenhada e sente-se realizado e satisfeito ${ }^{14}$.

Destarte, é primordial que o enfermeiro desenvolva conhecimento, habilidades e sensibilidade no relacionamento interpessoal, com base em suas próprias ações, constituindo uma interação pautada no encontro verdadeiro com os pacientes na finitude da vida, em que a intencionalidade do agir e a compreensão de cada profissional no processo de cuidar sejam manifestos ${ }^{15}$.

\section{Comunicação em cuidados paliativos como estratégia para fortalecimento do vínculo entre enfermeiro e paciente terminal}

A comunicação é um processo de envolvimento que deve ser constituir com o estabelecimento de vínculo entre o enfermeiro e o paciente terminal, de maneira verbal e não verbal. Logo, trata-se de um processo ativo, de atenção e de escuta ativa. Esse aspecto é referenciado pelos enfermeiros, conforme os relatos, a seguir:

Utilizo tanto a verbal, através da fala, como a não verbal por meio do olhar, de gestos, do toque, do carinho e do conforto. (Enf.21)

[...] sempre é importante verbalizar todas as ações realizadas previamente, transmitindo segurança, com empatia e otimismo. Comunicando procedimentos, elogiando a sua contribuição. (Enf.13)

Chegando perto do mesmo, muitas vezes segurando sua mão, conversando, olhando em seus olhos e deixando que ele fique a vontade para expressar seus sentimentos. (Enf.28)

[...] podendo-se estabelecer uma comunicação verbal ou não verbal, no intuito de atender a necessidade do paciente em todas as suas dimensões. (Enf.26)

Nas falas desses enfermeiros, é nítida a preocupação em atender às necessidades dos pacientes por meio da comunicação verbal e da não verbal. Eles destacam a importância do olhar, do toque, do carinho e do conforto, inseridos no universo do modo não verbal de se comunicar, na relação direta com o paciente terminal.

Assim sendo, a participação no cuidado de maneira verbal e não verbal, depende da abertura estabelecida entre as pessoas envolvidas, de forma que isso permita a sua proximidade no relacionamento existencial, e elas apresentem sua própria singularidade ${ }^{16}$.

Convém ressaltar que o emprego apropriado da comunicação verbal e não verbal é uma 
medida terapêutica eficaz para os pacientes terminais. É considerado um componente essencial do cuidado no fim da vida, uma vez que minimiza o estresse psicológico dos mesmos e lhe permite compartilhar o sofrimento. Assim, independentemente da capacidade de comunicação verbal do paciente, é dever do enfermeiro ouvi-lo e percebê-lo, identificando qual o estágio do processo de morrer em que se encontra e quais são suas necessidades, para orientar e capacitar sua equipe a suprir as demandas, possibilitando-lhes uma interação terapêutica, por meio da empatia e da criação de um ambiente saudável, humanizado e sistematizado ${ }^{11,17}$.

Observa-se que um dos aspetos da comunicação que influencia as estratégias da linguagem verbal pelos enfermeiros é o tom de voz, que deve ser firme e seguro quando necessário, como, por exemplo, para dar um diagnóstico, e doce o suficiente para se expressar numa situação de apoio psicológico ou um gesto de afeto. Portanto, a comunicação verbal é estabelecida por meio de palavras que expressam um pensamento, clarificam um fato ou validam a compreensão de algo, porém não é suficiente para caracterizar a complexa interação do que ocorre no relacionamento humano ${ }^{6}$. Destarte, é essencial ser acompanhada por emoções e sentimentos para que se possa compreender não só o significado próprio da palavra, mas também os sentimentos que vêm implícitos na mensagem. Tais fatos são realizados através da comunicação não verbal, por meio da qual é possível compreender os senti-mentos nos relacionamentos interpessoais ${ }^{11}$.

Por sua vez, a noção de que a comunicação não verbal permeia toda emissão verbal deve estar presente, uma vez que revela sentimentos e intenções, razão por que os sinais devem ser clarificados e questionados a fim se se compreender bem mais o momento vivido ${ }^{18}$.

Nesse sentido, ressalta-se que o estabelecimento de vínculo que é o objetivo do relacionamento interpessoal, requer a comunicação não verbal, imprescindível na relação entre o cuidador e o ser que é cuidado, que passam a confiar um no outro a partir da demonstração de empatia e de transmissão de segurança, por meio do olhar, do toque, dos gestos, das posturas corporais e do ouvir ${ }^{11}$.

A grandeza da escuta ativa, na prática dos cuidados paliativos, também foi considerada nos trechos dos depoentes:

É importante que o profissional sempre escute as preocupações dos pacientes e de sua família, seus anseios, suas dúvidas, seus desabafos, promovendo um cuidado com qualidade. (Enf.5)
[...] a questão de ouvir o outro é de suma relevância para entender as necessidades e anseios do paciente/cliente. (Enf.7)

Procuro ser empático, interagindo, acolhendo, ouvindo com calma e prudência, respeitando a vontade do paciente. (Enf.12)

[...] é importante acolher o paciente e seus familiares e fazer a escuta com qualidade, para assim fornecer segurança para eles. (Enf.27)

Os relatos mostraram que os enfermeiros, a partir de uma comunicação eficaz, demonstram preocupação e interesse pelos pacientes terminais e por sua família, escutando-os, buscando esclarecer dúvidas a respeito do estado de saúde e proporcionando-lhes segurança.

Vale salientar que uma das principais habilidades de comunicação necessária aos profissionais da Saúde é a escuta, que deve ser atenta e reflexiva, para que o profissional possa identificar as reais necessidades dos pacientes. Mostrarse disponível para ouvi-lo e compreendê-lo é uma maneira eficaz de ajudá-lo emocional e espiritualmente ${ }^{19}$. Escutar os pacientes terminais significa concentrar-se no paciente e em suas reais neces-sidades, em diferentes aspectos. Quando o profissional está preparado para a escuta, o paciente e seus familiares se sentem atendidos e satisfeitos em seus anseios e preocupações ${ }^{20}$.

Para tanto, é imprescindível que o enfermeiro transforme sua forma de assistir, passando do fazer para o escutar, perceber, compreender, identificar necessidades, para somente então, planejar ações. Nesse sentido, o escutar não é apenas ouvir, mas permanecer em silêncio, empregar gestos de afeto e sorriso que expressem aceitação e estimulem a expressão de sentimentos ${ }^{12}$.

Nessa perspectiva, os enfermeiros que trabalham em cuidados paliativos com pacientes em iminência de morte e com sua família devem valorizar o uso da comunicação verbal e da não verbal, bem como da escuta qualificada como instrumentos terapêuticos efetivos para promoção dessa modalidade de cuidar, embora, nem sempre, façam o uso delas. Tal fato corrobora os discursos de dois participantes do estudo: [...] apesar da importância da comunicação verbal e não verbal, estas são pouco praticadas no ambiente hospitalar. (Enf.19), [...] eu sei que é importante utilizar a verbalização com o paciente, utilizar o toque. Mas, é muito difícil eu fazer o uso dessas práticas, pois a rotina não permite. (Enf.16).

Desse modo, o serviço de educação continuada mostra-se de extrema relevância e indispensável para o treinamento e o constante aperfeiçoamento dos profissionais, mesmo os que já 
exercem atividades em instituições hospitalares com pacientes na finitude de vida.

\section{Importância da comunicação entre enfermeiro e família do paciente terminal sob cuidados paliativos}

Nesta categoria, os trechos dos depoimentos dos enfermeiros inseridos no estudo mencionaram a importância da comunicação como estratégia, no campo dos cuidados paliativos, direcionada aos familiares de pacientes em fase terminal, conforme evidenciado nos relatos a seguir:

A comunicação é um importante canal entre o paciente/família e a equipe, [...] é essencial para que haja um respeito mútuo e assim promover uma melhor qualidade de vida ao paciente e um melhor processo de luto aos familiares. (Enf.9)

A comunicação é fundamental para que todos da equipe de enfermagem assistam de maneira holistica e com maior sensibilidade. O carinho e o amor ao paciente e aos seus familiares são essenciais, pois os familiares fazem parte do processo da terminalidade. (Enf.14)

Durante a visita, sempre comunico ao familiar acerca da assistência de enfermagem prestada e do estado geral do paciente, dou um abraço no familiar e acolho da melhor maneira possivel, passando segurança e confiança para ele, procuro ter uma assistência humanizada (Enf.16)

[...] faz com que o paciente e o familiar ao se comunicar com a equipe tenha segurança e confiança acerca do serviço prestado, essa segurança deve ser passada para nós profissionais de enfermagem, que acabamos por manter um vínculo maior com o paciente. (Enf.18)

Os discursos desses enfermeiros ressaltam, de modo enfático, a importância da comunicação verbal e não verbal utilizada com a família do paciente em fase terminal. Esclareceram, ainda, que a família é o elo fundamental no processo de cuidado com o paciente e que, para se adquirir a confiança destes, é imprescindível uma boa interação entre os profissionais, pacientes e familiares.

Observa-se que a comunicação com o paciente e com os membros da família é primordial para que os profissionais da saúde, em essencial o enfermeiros, possam oferecer um serviço de qualidade, pois somente através de uma comunicação efetiva com todos os membros é que ele estará apto a incluí-la adequadamente na terapêutica dos cuidados paliativos ${ }^{21}$.

Com base em tal entendimento, o estabelecimento de uma comunicação efetiva entre a tríade enfermeiro-paciente-família é sobremaneira relevante, para se conhecer bem mais o paciente e suas necessidades, com habilidade e compreensão, e proporcionar-lhe uma assistência terapêutica singular. Neste prisma, o diálogo entre o profissional da Saúde e a família do paciente terminal pode desvendar muitos anseios, medos e dúvidas presentes nessa dimensão e, de tal modo, promover a criação de vínculo, importante e necessário nessa etapa da vida ${ }^{18}$.

A questão da segurança e da confiança é explanada nos relatos dos profissionais (Enf. 16 e Enf. 18), que referem os seus cuidados consubstanciados em uma abordagem humanizada, que é permeada por uma relação legítima com os familiares, mais próximos deles. Essa relação ocorre pelo fato de os enfermeiros passarem a maior parte do tempo dispensando sua assistência ao paciente e a sua família, estabelecendo uma relação de amizade e, consequentemente, desenvolvendo um vínculo maior ${ }^{22}$.

Ante o exposto, ressalta-se que a comunicação (verbal e não verbal) foi compreendida pelos enfermeiros envolvidos no estudo como parte essencial do cuidado com o paciente terminal e aos seus familiares. A relevância do tipo de linguagem utilizada depende do grau de comprometimento do paciente, porém é possível se comunicar passando informações que confortam, esclarecem e dignificam a finitude humana. Logo, o relacionamento interpessoal que ocorre entre o enfermeiro e o paciente/familiar, no processo do cuidar, tem sua essência nas habilidades de comunicação, e isso é fundamental para que a assistência de enfermagem seja humanizada.

\section{Considerações Finais}

O presente estudo, por meio do qual se abordou, com base na filosofia dos cuidados paliativos, a comunicação entre os enfermeiros e o paciente terminal, ressalta a importância de um cuidar centrado no paciente em sua totalidade, $\mathrm{e}$ não, apenas, em sua doença.

Ficou evidente, por meio dos depoimentos dos enfermeiros participantes do estudo, que estes consideram o relacionamento interpessoal com o paciente em fase terminal e com seus familiares como uma ferramenta imprescindível para a promoção dos cuidados paliativos nessa etapa de vida, haja vista que lhes permite esclarecer dúvidas, através de uma linguagem verbal e não verbal que seja simples e acessível, de forma que os pacientes exteriorizem suas angústias e 
seus medos. Outro ponto que merece destaque diz respeito ao reconhecimento, por alguns enfermeiros envolvidos na pesquisa, quanto à participação da família no processo de cuidar do paciente em fase terminal.

Nesse sentido, a pesquisa evidenciou a relevância da comunicação como uma estratégia fundamental para respaldar a prática clínica do enfermeiro direcionada ao paciente em fase terminal. Entretanto, acredita-se ser necessária a reaplicação deste estudo com os pacientes na terminalidade e sua família para confirmarem a relevância da comunicação como uma estratégia fundamental para respaldar a prática dos cuidados paliativos direcionada ao paciente na finitude da vida.

\section{Colaboradores}

CG Andrade trabalhou na análise e interpretação dos dados e na redação do artigo. SFG Costa trabalhou na revisão crítica e na aprovação da versão a ser publicada. MEL Lopes trabalhou na revisão crítica e na aprovação da versão a ser publicada. 


\section{Referências}

1. Santos FS. O desenvolvimento histórico dos cuidados paliativos e a filosofia hospice. In: Santos FS, organizador. Cuidados paliativos: diretrizes, humanização e alívio dos sintomas. São Paulo: Atheneu; 2011. p. 3-15.

2. Floriani CA, Schramm FR. Cuidados paliativos: interfaces, conflitos e necessidades. Cien Saude Colet 2008; 13(Supl. 2):2123-2132.

3. Matsumoto DY. Cuidados paliativos: conceito, fundamentos e princípios. In: Carvalho RT, Parsons HA. Manual de cuidados paliativos ANCP. 2a Edição. Porto Alegre: Sulina; 2012. p. 23-41.

4. Correia FR, Carlo MMRP. Avaliação de qualidade de vida no contexto dos cuidados paliativos: revisão integrativa de literatura. Rev Lat Am Enfermagem 2012; 20(2):401-410.

5. Mullan BA, Kothe EJ. Evaluating a nursing communication skills training course: the relationships between self-rated ability, satisfaction, and actual performance. Nurse Educ Pract 2010; 10(6):374-378.

6. Rodrigues MVC, Ferreira ED, Menezes TMO. Comunicação da enfermeira com pacientes portadores de câncer fora de possibilidade de cura. Rev Enferm UERJ 2010; 18(1):86-91.

7. Oliveira AC, Silva MJP. Autonomia em cuidados paliativos: conceitos e percepções de uma equipe de saúde. Acta Paul Enferm 2010; 23(2):212-217.

8. Brasil. Ministério da Saúde (MS). Conselho Nacional de Saúde. Resolução n. 196, de 10 de outubro de 1996. Diretrizes e normas regulamentadoras de pesquisa envolvendo seres humanos. Diário Oficial da União 1996; 16 out.

9. Bardin L. Análise de conteúdo. São Paulo: Edições $70 ; 2011$.

10. Jaccobsen J, Jackson VA. A communication approach for oncologists: understanding patient coping and communicating about bad news, palliative care, and hospice. J Natl Compr Canc Netw 2009; 7(4):475480.

11. Araújo MMT, Silva MJPA. Estratégias de comunicação utilizadas por profissionais de saúde na atenção à pacientes sob cuidados paliativos. Rev Esc Enferm USP 2012: 46(3):626-632.

12. Araújo MMT, Silva MJP. A comunicação com o paciente em cuidados paliativos: valorizando a alegria e o otimismo. Rev Esc Enferm USP 2007; 41(4):668-674.
13. Buisán R, Delgado JC. El cuidado del paciente terminal. An Sist Sanit Navar 2007; 30(Supl.3):103-112.

14. Tomlinson D, Capra M, Gammon J, Volpe J, Barrera M, Hinds PS, et al. Parental decision making in pediatric cancer end-of-life care: using focus group methodology as a prephase to seek participant design input. Eur J Oncol Nurs 2006; 10(3):198-206.

15. Theunissen JMJ, Hoogerbrugge PM, Van Achterberg T, Prins JB, Vernooij-Dassen MJFJ, Van DenEnde CHM. Symptoms in the palliative phase of children with cancer. Pediatr Blood Cancer 2007; 49:160-165.

16. Chico CE, Nascimento EC, Castanheira L, Lima RAG. Children and adolescents with cancer: experiences with chemotherapy. Rev Lat Am Enfermagem 2010;18(5):864-872.

17. Foschiera F, Piccoli M. Enfermagem perioperatória: diagnósticos de enfermagem emocionais e sociais. Cienc Cuid Saude 2004; 3(2):143-151.

18. Ordahi LFB, Padilha MICS, Souza LNAS. Comunicação entre a enfermagem e os clientes impossibilitados de comunicação verbal. Rev Lat Am Enfermagem 2007; 15(5):85-93.

19. Silva MJP, Araújo MMT. Comunicação em cuidados paliativos. In: Carvalho RT, Parsons HA. $M a$ nual de cuidados paliativos ANCP. 2a Edição. Porto Alegre: Sulina; 2012. p.75-85.

20. Higuera JCB. La escucha activa em cuidados paliativos. Rev Est Med Hum 2005; 11(11):119-136.

21. Lima AC, Silva JAS, Silva MJP. Profissionais de saúde, cuidados paliativos e família: revisão bibliográfica. Cogitare Enferm 2009; 14(2):360-367.

22. Morais GSN, Costa SFG, Fontes WD, Carneiro AD. Comunicação como instrumento básico no cuidar humanizado em enfermagem ao paciente hospitalizado. Acta Paul Enferm 2009; 22(3):323-327.

Artigo apresentado em 29/04/2013

Aprovado em 22/05/2013

Versão final apresentada em 27/05/2013 